\title{
THE ARITHMETIZED EXPANSIONS FOR CERTAIN DOUBLY PERIODIC FUNCTIONS OF THE THIRD KIND*
}

BY G. D. NICHOLS

1. Introduction. In this paper the explicit arithmetized Fourier developments are given for certain doubly periodic functions of the third kind, namely,

$$
\begin{aligned}
& \Phi_{\alpha \beta \gamma}(x, y)=\vartheta_{1}^{\prime} \vartheta_{\alpha}(x+y) / \vartheta_{\beta}^{3}(x) \vartheta_{\gamma}(y), \\
& \Psi_{\alpha \beta \gamma}(x, y)=\vartheta_{1}^{\prime 3} \vartheta_{\alpha}^{2}(x+y) / \vartheta_{\beta}{ }^{3}(x) \vartheta_{\gamma}^{2}(y),
\end{aligned}
$$

where $x$ and $y$ are independent complex variables, the $\vartheta$ 's are the Jacobi theta functions, and $\alpha, \beta, \gamma$ are a certain sixteen triads out of the sixty-four which can be selected from the numbers $0,1,2,3$. The method of obtaining the expansions is essentially that used by Basoco $†$ in obtaining the arithmetized developments for certain functions similar in form.

The applications to arithmetic lead to arithmetical paraphrase equations involving incomplete numerical functions in two variables. The complexity of the expansions is not adverse to simple and interesting arithmetical results, since simplicity can be gained by specialization while the complexity gives greater variety.

There are thirty-two expansions given in all. The domain of validity for $x$ is $-I(\pi \tau)<I(x)<I(\pi \tau)$ in (1)-(8) and (17)-(24) inclusive, and $-(1 / 2) I(\pi \tau)<I(x)<(1 / 2) I(\pi \tau)$ for $(9)-(16)$ and (25)-(32) inclusive; $y$ is restricted in all expansions to $-(1 / 2) I(\pi \tau)<I(y)<(1 / 2) I(\pi \tau)$.

In all the series the outer summation refers to positive nonzero integers $m, n, \alpha$ in the exponent of $q$, and extends to all $m=1,3,5, \cdots$, to all $n=1,2,3, \cdots$, or $2,4,6, \cdots$, to all $\alpha=1,5,9, \ldots$. The coefficient of the power of $q$ is in parentheses after the power. The inner summation is finite and refers to positive integral divisors $t, \tau, d, \delta$, of $m, n, \alpha$, subject to further restrictions noted below. The integer $\tau$ is always odd. The

* Presented to the Society, November 30, 1934.

$\dagger$ American Journal of Mathematics, vol. 54 (1932), p. 242. 
values and necessary arithmetized expansions for the coefficients $A_{i}, B_{i}, C_{i},(i=0,1,2,3)$, are given below in $\$ 4$.

2. The Functions $\Phi_{\alpha \beta \gamma}(x, y)$. In this section $N 1, N 2, \cdots$, where $1,2, \cdots$ refer to the serial number of the expansion, are equal to the expression in brackets following $A_{i} / 2$. The symbol $\mathcal{D}$ is the operator $(\partial / \partial x+2 \partial / \partial y)$.

In the expansions (1)-(4) inclusive, we have $n=d \delta, d \leqq n^{1 / 2}$, $z=2(\delta-d) x+2 d y$. When $d=n^{1 / 2}$ the value obtained from the inner summation is to be divided by 2 .

$$
\text { (1) } \begin{aligned}
\Phi_{111}(x, y)= & (1 / 2) A_{1}\left[\operatorname{ctn} x+4 \sum q^{2 n}\left(\sum \sin z\right)\right] \\
& -C_{1} \mathcal{D}(N 1)+(1 / 2) \mathcal{D}^{2}(N 1), \\
(2) \quad \Phi_{221}(x, y)= & (1 / 2) A_{1}\left[-\tan x+4 \sum q^{2 n}\left(\sum(-1)^{\delta-d} \sin z\right)\right] \\
& -C_{1} \mathcal{D}(N 2)+(1 / 2) \mathscr{D}^{2}(N 2), \\
(3) \quad \Phi_{212}(x, y)= & (1 / 2) A_{2}\left[\operatorname{ctn} x+4 \sum q^{2 n}\left(\sum(-1)^{d} \sin z\right)\right] \\
& -C_{2} \mathcal{D}(N 3)+(1 / 2) \mathscr{D}^{2}(N 3), \\
\text { (4) } \quad \Phi_{122}(x, y)= & (1 / 2) A_{2}\left[\tan x-4 \sum q^{2 n}\left(\sum(-1)^{\delta} \sin z\right)\right] \\
& -C_{2} \mathcal{D}(N 4)+(1 / 2) \mathcal{D}^{2}(N 4) .
\end{aligned}
$$

In (5)-(8) inclusive $n=t \tau, t<(n / 2)^{1 / 2}, z=(\tau-2 t) x+2 t y$.

(6) $\Phi_{320}(x, y)=(1 / 2) A_{0}\left[\sec x+4 \sum q^{n}\left(\sum(-1 / \tau-2 t) \cos z\right)\right]$

$$
-C_{0} \mathcal{D}(N 6)+(1 / 2) \mathcal{D}^{2}(N 6) \text {, }
$$

$$
\begin{aligned}
\Phi_{313}(x, y)= & (1 / 2) A_{3}\left[\csc x+4 \sum q^{n}\left(\sum(-1)^{t} \sin z\right)\right] \\
& -C_{3} \mathcal{D}(N 7)+(1 / 2) \mathcal{D}^{2}(N 7), \\
\Phi_{023}(x, y)= & (1 / 2) A_{3}\left[\sec x+4 \sum q^{n}\left(\sum(-1 / \tau) \cos z\right)\right] \\
& -C_{3} \mathcal{D}(N 8)+(1 / 2) \mathcal{D}^{2}(N 8) .
\end{aligned}
$$

In (9)-(12) inclusive $m=t \tau, \tau \leqq m^{1 / 2}, z=(t-\tau) x+\tau y$. When $\tau=m^{1 / 2}$ the value obtained from the inner summation is to be divided by 2 .

$$
\begin{aligned}
\Phi_{001}(x, y)= & (1 / 2) A_{1}\left[-4 \sum q^{m / 2}\left(\sum \sin z\right)\right] \\
& -C_{1} \mathscr{D}(N 9)+(1 / 2) \mathcal{D}^{2}(N 9),
\end{aligned}
$$


(10)

$$
\begin{aligned}
\text { (10) } \Phi_{331}(x, y)= & (1 / 2) A_{1}\left[-4 \sum q^{m / 2}\left(\sum(-1)^{(t-\tau) / 2} \sin z\right)\right] \\
& -C_{1} \mathcal{D}(N 10)+(1 / 2) \mathcal{D}^{2}(N 10), \\
\text { (11) } \Phi_{302}(x, y)= & (1 / 2) A_{2}\left[-4 \sum q^{m / 2}\left(\sum(-1 / \tau) \cos z\right)\right] \\
& -C_{2} \mathcal{D}(N 11)+(1 / 2) \mathcal{D}^{2}(N 11), \\
\text { (12) } \quad \Phi_{032}(x, y)= & (1 / 2) A_{2}\left[4 \sum q^{m / 2}\left(\sum(-1 / \tau) \cos z\right)\right] \\
& -C_{2} \mathcal{D}(N 12)+(1 / 2) \mathcal{D}^{2}(N 12) .
\end{aligned}
$$

In (13)-(16) inclusive $n=t \tau, \tau<n^{1 / 2}, z=(t-\tau) x+\tau y, t$ assuming even positive integral values only.

$$
\begin{aligned}
(13) \Phi_{203}(x, y)= & (1 / 2) A_{3}\left[-4 \sum q^{n / 2}\left(\sum(-1 / \tau) \cos z\right)\right] \\
- & C_{3} \mathcal{D}(N 13)+(1 / 2) \mathcal{D}^{2}(N 13), \\
(14) \quad \Phi_{133}(x, y)= & (1 / 2) A_{3}\left[4 \sum q^{n / 2}\left(\sum(-1)^{t / 2} \sin z\right)\right] \\
& -C_{3} \mathcal{D}(N 14)+(1 / 2) \mathcal{D}^{2}(N 14), \\
(15) \quad \Phi_{100}(x, y)= & (1 / 2) A_{0}\left[-4 \sum q^{n / 2}\left(\sum \sin z\right)\right] \\
& -C_{0} \mathcal{D}(N 15)+(1 / 2) \mathcal{D}^{2}(N 15), \\
(16) \quad \Phi_{230}(x, y)= & (1 / 2) A_{0}\left[4 \sum q^{n / 2}\left(\sum(-1 / \tau+t) \cos z\right)\right] \\
& -C_{0} \mathcal{D}(N 16)+(1 / 2) \mathcal{D}^{2}(N 16) .
\end{aligned}
$$

3. The Functions $\Psi_{\alpha \beta \gamma}(x, y)$. Here $N 17, N 18, \cdots$ are equal to the expressions in brackets following $B_{i}, \mathcal{D}$ is the operator $(2 \partial / \partial x+\partial / \partial y)$. The substitution $(i, j)$ is one which replaces $B_{i}$ and $C_{i}$ in the right member of any expansion by $B_{j}$ and $C_{j}$, respectively.

In (17)-(24) inclusive $n=d \delta, \delta-d \equiv 1(\bmod 2), d<n^{1 / 2}$, $z=(\delta-d) x+4 d y$.

(17) $\Psi_{111}(x, y)=B_{1}\left[\csc x+4 \sum q^{n}\left(\sum(-1)^{d} \sin z\right)\right]$

$$
-C_{1} \mathcal{D}(N 17)+(1 / 8) \mathcal{D}^{2}(N 17) \text {, }
$$

(18) $\Psi_{221}(x, y)=B_{1}\left[\sec x+4 \sum q^{n}\left(\sum(-1 / d+\delta) \cos z\right)\right]$

$$
-C_{1} \mathcal{D}(N 18)+(1 / 8) \mathcal{D}^{2}(N 18) \text {, }
$$

(19) $\Psi_{212}(x, y)$ is written from (17) by the substitution $(1,2)$,

(20) $\Psi_{122}(x, y)$ is written from (18) by the substitution $(1,2)$,

(21) $\Psi_{010}(x, y)$ is written from (17) by the substitution $(1,0)$,

(22) $\Psi_{320}(x, y)$ is written from (18) by the substitution $(1,0)$, 
(23) $\Psi_{313}(x, y)$ is written from (17) by the substitution $(1,3)$,

(24) $\Psi_{023}(x, y)$ is written from (18) by the substitution $(1,3)$.

In the formulas (25)-(32) inclusive $\alpha=t \tau, \alpha \equiv 1(\bmod 4)$, $\tau \leqq \alpha^{1 / 2}, z=((t-\tau) / 2) x+2 \tau y$. When $\tau=\alpha^{1 / 2}$ the value obtained from the inner summation is to be divided by 2 .

$$
\begin{aligned}
\Psi_{001}(x, y)= & B_{1}\left[4 \sum q^{\alpha / 4}\left(\sum(-1 / \tau) \cos z\right)\right] \\
& -C_{1} \mathcal{D}(N 25)+(1 / 8) \mathcal{D}^{2}(N 25), \\
\Psi_{331}(x, y)= & B_{1}\left[4 \sum q^{\alpha / 4}\left(\sum(-1)^{(t+\tau-2) / 4} \cos z\right)\right] \\
& -C_{1} \mathcal{D}(N 26)+(1 / 8) \mathcal{D}^{2}(N 26),
\end{aligned}
$$

(27) $\Psi_{302}(x, y)$ is written from $-\Psi_{001}(x, y)$ by the substitution $(1,2)$,

(28) $\Psi_{032}(x, y)$ is written from $-\Psi_{331}(x, y)$ by the substitution $(1,2)$,

(29) $\Psi_{203}(x, y)$ is written from $-\Psi_{001}(x, y)$ by the substitution $(1,3)$,

(30) $\quad \Psi_{133}(x, y)$ is written from $-\Psi_{331}(x, y)$ by the substitution $(1,3)$,

(31) $\Psi_{100}(x, y)$ is written from (25) by the substitution $(1,0)$,

(32) $\Psi_{230}(x, y)$ is written from (26) by the substitution $(1,0)$.

4. Arithmetization of the Coefficients. The values of the coefficients are

$$
A_{i}=\frac{\vartheta_{i}{ }^{\prime \prime}(y)}{\vartheta_{i}(y)}-\frac{\vartheta_{1}{ }^{\prime \prime \prime}}{\vartheta_{1}{ }^{\prime}}, \quad B_{i}=\frac{\vartheta_{i}{ }^{\prime \prime}(y)}{\vartheta_{i}(y)}+\frac{\vartheta_{i}{ }^{\prime 2}(y)}{\vartheta_{i}{ }^{2}(y)}-\frac{\vartheta_{1}{ }^{\prime \prime \prime}}{2 \vartheta_{1}{ }^{\prime}}, \quad C_{i}=\frac{\vartheta_{i}{ }^{\prime}(y)}{\vartheta_{i} y} .
$$

The necessary arithmetized expansions for the $C_{i}$ 's are given in a list by Bell.* In the following expansions for the others, two types of divisors occur, namely, $n=d \delta$, where $d$ is arbitrary and $\delta$ even, and $n=t \tau$, where $t$ is arbitrary and $\tau$ odd.

(a) $A_{0}=1+8 \sum q^{n}\left(\sum \tau \cos 2 t y\right)-8 \sum q^{n}\left(\sum \delta\right)$,

(b) $A_{1}=8 \sum q^{n}\left(\sum \delta \cos 2 d y\right)-8 \sum q^{n}\left(\sum \delta\right)$,

* Messenger of Mathematics, vol. 53 (1924), p. 166. 
(c) $\quad A_{2}=8 \sum q^{n}\left(\sum(-1)^{d} \delta \cos 2 d y\right)-8 \sum q^{n}\left(\sum \delta\right)$,

(d) $A_{3}=1+8 \sum q^{n}\left(\sum(-1)^{t} \tau \cos 2 t y\right)-8 \sum q^{n}\left(\sum \delta\right)$,

(e) $\quad B_{0}=1 / 2+2 \sum q^{n}\left(\sum \delta\right)+8 \sum q^{n}\left(\sum(2 \tau-t) \cos 2 t y\right)$,

(f) $\quad B_{1}=-3 / 2+\csc ^{2} y+2 \sum q^{n}\left(\sum \delta\right)$

$$
+8 \sum q^{n}\left(\sum(2 \delta-d) \cos 2 d y\right) \text {, }
$$

(g) $\quad B_{2}=-3 / 2+\sec ^{2} y+2 \sum q^{n}\left(\sum \delta\right)$

$$
+8 \sum q^{n}\left(\sum(2 \delta-d)(-1)^{d} \cos 2 d y\right)
$$

(h) $\quad B_{3}=1 / 2+2 \sum q^{n}\left(\sum \delta\right)$

$$
+8 \sum q^{n}\left(\sum(-1)^{t}(2 \tau-t) \cos 2 t y\right) .
$$

UNIVERSITY OF ARKANSAS

\section{A CONNECTEDNESS THEOREM IN ABSTRACT SETS*}

BY W. M. WHYBURN

This note gives a variation of a theorem of Sierpinski and Saks. $\dagger$ The theorem is valid in spaces which have the BorelLebesgue property (Axiom I of Saks $\ddagger$ ) and which satisfy axioms (A), (B), (C), and (6) as given by Hausdorff. $\$$ We use the term connected for a closed set to mean that the set cannot be expressed as the sum of two mutually exclusive non-vacuous, closed sets.||

TheOREM. Let $F$ be a collection of closed sets at least one of which is compact. Let $F$ contain more than one element and let it be true that the sets of each finite sub-collection of $F$ have a nonvacuous, connected set in common when this sub-collection contains at least two elements of $F$. Under these hypotheses, there is a closed, non-vacuous, connected set common to all of the sets of collection $F$.

Proof. Let $F_{0}$ be a compact member of collection $F$ and let $K$ be the set of points common to all of the sets of collection $F$.

* Presented to the Society, December 1, 1934.

$\dagger$ See Saks, Fundamenta Mathematicae, vol. 2 (1921), pp. 1-3.

¥ Saks, ibid., p. 2.

\$ Mengenlehre, 1927, pp. 228-229.

|| The notion of limit point may be defined and this definition used to describe connectedness. We use domain and open set interchangeably. 